\title{
Profil Clinique et Radiologique des Spondylolisthesis Lombaires Vus au Centre Hospitalier Universitaire de Brazzaville
}

\section{Lamini N'soundhat N. E.,}

Service de Rhumatologie,

Centre Hospitalier Universitaire de Brazzaville, République du Congo

Boukassa L.,

Service de Neurochirurgie,

Centre Hospitalier Universitaire de Brazzaville, République du Congo

Ngampika Tsiba E. F.,

Service de Rhumatologie,

Centre Hospitalier Universitaire de Brazzaville, République du Congo.

Moyikoua R. F.,

Service d'Imagerie Médicale,

Centre Hospitalier Universitaire de Brazzaville, République du Congo

Ntsiba H.,

Service de Rhumatologie,

Centre Hospitalier Universitaire de Brazzaville, République du Congo

Bileckot $\boldsymbol{R}$.,

Service d'Immuno-Rhumatologie et de Rééducation Fonctionnelle,

Centre Hospitalier Universitaire de Brazzaville, République du Congo

Doi:10.19044/esj.2019.v15n30p413 URL:http://dx.doi.org/10.19044/esj.2019.v15n30p413

\section{Resume}

Objectif: Décrire le profil clinique et radiologique des spondylolisthésis lombaires vus en milieu hospitalier. Patients et Methode: Etude descriptive, sur dossiers médicaux, menée dans les services de Rhumatologie et de Neurochirurgie du CHU de Brazzaville (Congo), du $1^{\text {er }}$ janvier 2008 au 31 décembre 2018 soit 11 ans. Ont été inclus : les patients vus pour lombalgie / lomboradiculalgie commune, dont le dossier comportait une imagerie du rachis lombaire affirmant le spondylolisthésis (radiographie standard/ Tomodensitométrie/IRM). Resultats: Trois cent soixante onze dossiers colligés parmi 2961, soit une fréquence hospitalière de 13,2\%. Il s'agissait de 46 hommes $(12,4 \%)$ et 325 femmes $(87,6 \%)$ soit un sex-ratio de 0,1 . L'âge moyen était de 61,2 $\pm 9,8$ ans (extrêmes 28 et 89 ans). La durée moyenne d'évolution était de 2,3 $\pm 2,6$ ans (extrêmes 1 mois à 11 ans). La 
douleur était le maître symptôme, à type de lombalgie dans 99,2\% des cas, associée à une radiculalgie dans $70,9 \%$ des cas. Elle était unilatérale dans $52,5 \%$ et bilatérale dans $47,5 \%$ des cas, de topographie L5 (79,8\%), S1 $(13,7 \%)$, L4 $(5,3 \%)$ et L3 $(1,1 \%)$. Une claudication neurologique était retrouvée dans $63,3 \%$ des cas. Le spondylolisthésis siégeait à l'étage L4-L5 dans $72,8 \%$ des cas, L5-S1 dans 15,9\% et L3-L4 dans 11,3\%. Le spondylolisthésis était de grade I dans $71,4 \%$ et de grade II dans $28,6 \%$ des cas. Il était de type dégénératif dans $91,6 \%$ des cas et de type isthmique 8,4\% des cas. Conclusion : Affection de l'adulte d'âge mûr, à prédominance féminine. Il siège aux étages L4-L5 et L5-S1, de grade I et II exclusivement. L'étiologie dégénérative en est la principale cause.

Mots clés : Spondylolisthésis, Spondylolyse, Lomboradiculalgie, Lombalgie 


\title{
Clinical and Radiological Profile of Lombar Spondylolisthesis at the University Teaching Hospital of Brazzaville
}

\section{Lamini N'soundhat N. E.,}

Service de Rhumatologie,

Centre Hospitalier Universitaire de Brazzaville, République du Congo

\section{Boukassa L.,}

Service de Neurochirurgie,

Centre Hospitalier Universitaire de Brazzaville, République du Congo

Ngampika Tsiba E. F.,

Service de Rhumatologie,

Centre Hospitalier Universitaire de Brazzaville, République du Congo.

Moyikoua R. F.,

Service d'Imagerie Médicale,

Centre Hospitalier Universitaire de Brazzaville, République du Congo

Ntsiba $\mathrm{H}$.,

Service de Rhumatologie,

Centre Hospitalier Universitaire de Brazzaville, République du Congo

\section{Bileckot R.,}

Service d'Immuno-Rhumatologie et de Rééducation Fonctionnelle, Centre Hospitalier Universitaire de Brazzaville, République du Congo

\begin{abstract}
Objective: To describe the clinical and radiological profile of lumbar spondylolisthesis seen in a hospital setting. Patients and method: A crosssectional survey, on medical records, conducted in the departments of Rheumatology and Neurosurgery of the University Teaching Hospital of Brazzaville (Congo), from January 1, 2008 to December 31, 2018 or 11 years. Included were: patients seen for low back pain / common lomboradiculalgia, whose record included lumbar spine imaging confirming the spondylolisthesis. (standard X-ray / CT / MRI). Results: Three hundred seventy-one files collected among 2961, showing a hospital frequency of $13.2 \%$. It was 46 men (12.4\%) and 325 women (87.6\%), a sex ratio of 0.1 . The mean age was $61.2 \pm 9.8$ years (range 28 to 89 years). Mean duration of evolution was $2.3 \pm 2.6$ years ( 1 month to 11 years). Pain was the main symptom, with low back pain in $99.2 \%$ of cases, associated with radiculalgia
\end{abstract}


in $70.9 \%$ of cases. It was unilateral in $52.5 \%$ and bilateral in $47.5 \%$ of cases, topography L5 (79.8\%), S1 (13.7\%), L4 (5.3\%) and L3 (1.1\%). Neurological claudication was found in $63.3 \%$ of cases. The spondylolisthesis was localized in L4-L5 in $72.8 \%$ of cases, L5-S1 in $15.9 \%$ and L3-L4 in $11.3 \%$. The spondylolisthesis was grade I in $71.4 \%$ and grade II in $28.6 \%$ of cases. It was degenerative in $91.6 \%$ of cases and isthmic in $8.4 \%$ of cases. Conclusion: Affection of the mature adult, predominantly female. He sits on floors L4-L5 and L5-S1, grade I and II exclusively. The degenerative etiology is the main cause.

Keywords: Spondylolisthesis, Spondylolysis, Lomboradiculalgia, Low back pain

\section{Introduction}

Le spondylolisthésis (SPL) est une cause fréquente de lombalgie et lomboradiculalgie communes, parfois invalidantes. Décrit pour la première fois par Herbiniaux en 1782 (Herbiniaux G, 1782), le spondylolisthésis se définit comme le glissement antérieur permanent du corps vertébral, accompagné des pédicules, des processus transverses et des articulaires postérieures, par rapport à la vertèbre sous-jacente (Vital J M et al, 2005). L'importance du déplacement est appréciée par la classification Meyerding (Meyerding $\mathrm{H} \mathrm{W}$ et al, 1932). On distingue 5 types de spondylolisthésis selon les travaux de Wiltse, à savoir: le type I ou type dysplasique, le type II ou isthmique, le type III ou dégénératif, le type IV ou traumatique et enfin le type V ou pathologique (Wiltse L L et al, 1976). Deux formes prédominent, le type II ou spondylolyse et le type III ou dégénératif. Le spondylolisthésis lombaire est une affection ubiquitaire touchant l'enfant à partir de l'âge scolaire (fréquence de 4-5\% vers 5-7 ans dans la population caucasienne) et l'adulte (Boisaubert $\mathrm{B}$ et al, 2001). Sa prévalence est estimée à $6 \%$ chez les européens, $15 \%$ chez les asiatiques et esquimaux (Dufor $X$ et al, 2013). En Afrique subsaharienne, les études sur le spondylolisthésis lombaire sont peu nombreuses (Varlet G et al, 1997; Amadou A et al, 2017; Iba Ba J et al, 2006). Au Congo, aucune étude n'a été menée sur cette affection alors qu'elle représente $15 \%$ des causes de lombalgie commune (Ntsiba $\mathrm{H}$ et al, 2009). L'objectif de cette étude a été de décrire le profil clinique et radiologique des spondylolisthésis en milieu hospitalier.

\section{Patients et Methodes}

Cette étude descriptive, transversale a été menée dans les services de Rhumatologie et de Neurochirurgie du Centre Hospitalier Universitaire de Brazzaville (Congo). Les dossiers médicaux des patients suivis pour un spondylolisthésis lombaire ont été colligés de la période allant du $1^{\mathrm{er}}$ janvier 
2008 au 31 décembre 2018, soit 11 ans. Ont été inclus, tous les dossiers de patients vus pour lombalgie / lomboradiculalgie commune ou syndrome du canal lombaire rétréci et dont le dossier comportait une imagerie du rachis lombaire affirmant le SPL, à savoir : une radiographie standard d'incidence de face et de profil et/ ou une tomodensitométrie (TDM) en coupe axiale et sagittale, sans injection de produit de contraste avec fenêtre osseuse et parenchymateuse et/ou une Imagerie par résonance magnétique (IRM), sans injection de produit de contraste, en séquence T1, T2, STIR. Les variables d'étude comportaient les données épidémiologiques (âge, sexe, profession), cliniques (circonstance de découverte, délai diagnostic, pratique sportive, les signes du syndrome rachidien lombaire, du syndrome radiculaire et du syndrome neurologique), diagnostiques (le siège du SPL, le stade radiographique selon la classification de Meyerding (Meyerding $\mathrm{H} \mathrm{W}$ et al, 1932), le type de spondylolisthésis selon la classification de Wiltse (Wiltse L $\mathrm{L}$ et al, 1976). Les données ont été colligées à l'aide d'une fiche d'enquête individuelle, dans le respect de l'anonymat et de la confidentialité. Les données morphologiques étaient interprétées par un radiologue et un rhumatologue afin de réduire le biais d'interprétation. Lorsque le patient présentait plusieurs pathologies, seul les éléments en rapport avec le SPL étaient pris en compte et rapportés sur la fiche d'enquête. Pour les patients hospitalisés à plusieurs reprises dans les services, seule la première admission a été prise en compte. La saisie, l'analyse et le traitement des données ont été réalisés à l'aide des logiciels CSPRO version 7.2 et SPSS 22. Les différents tableaux et graphiques ont été générés grâce au logiciel Microsoft Office Excel 2016.

\section{Resultats}

Pendant la période d'étude, 2961 patients ont été suivis dont 393 l'ont été pour un SPL. Parmi-eux, seuls 371 dossiers répondaient aux critères d'inclusion, soit une fréquence hospitalière de 13,2\%. Notre population d'étude comptait 46 hommes (12,4\%) et 325 femmes $(87,6 \%)$ soit un sex-ratio (H/F) de 0,1. L'âge moyen était de 61,2 $\pm 9,8$ ans avec des extrêmes de 28 et 89 ans. Le SPL prédominait entre 50 et 60 ans chez l'homme et entre 60 et 70 ans chez la femme. La répartition des patients selon l'âge et le genre est présentée dans la figure 1 . 


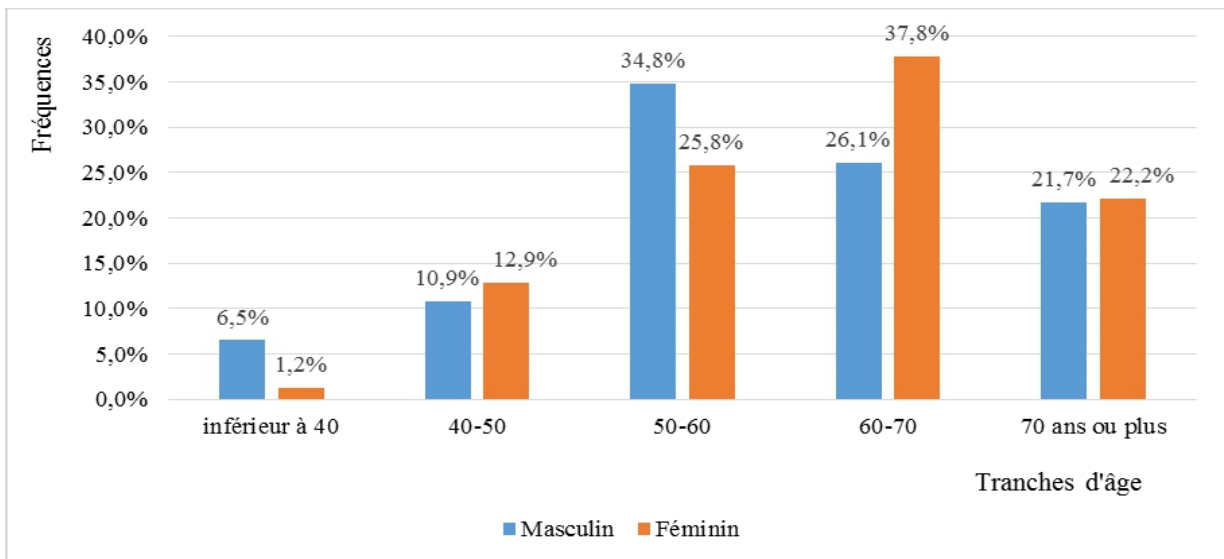

Figure 1 : Répartition des patients en fonction de l'âge et du genre

Les femmes au foyer étaient les plus touchés 93 cas $(25,16 \%)$, suivis des professionnels actifs et des retraités avec respectivement 81 cas $(21,8 \%)$ et 76 cas $(20,5 \%)$ (Tableau 1$)$.

Tableau I : Répartition des patients en fonction du statut socio-professionnel

\begin{tabular}{lcc}
\hline Statut socio-professionnel & Effectifs & Fréquences $\mathbf{( \% )}$ \\
\hline Ménagère & 93 & 25,1 \\
Professionnel actif & 81 & 21,8 \\
Retraité & 76 & 20,5 \\
Cultivateur & 39 & 10,5 \\
Sans emploi & 37 & 10 \\
Commerçant & 33 & 8,9 \\
Travailleur de force & 11 & 3 \\
Elève/Etudiant & 1 & 0,2 \\
\hline Total & $\mathbf{3 7 1}$ & $\mathbf{1 0 0}$ \\
\hline
\end{tabular}

Seuls 24 patients $(6,5 \%)$ pratiquaient une activité sportive, principalement le jogging (20 cas). La durée moyenne d'évolution du SPL avant le diagnostic était de 2,3 $\pm 2,6$ ans avec des extrêmes allant de 1 mois à 11 ans. La douleur était le maître symptôme, retrouvée chez tous les patients. Il s'agissait d'une lombalgie dans 368 cas $(99,2 \%)$ associée à une radiculalgie dans 263 cas (70,9\%). La radiculalgie était unilatérale dans 138 cas (52,5\%) et bilatérale dans 125 cas $(47,5 \%)$. La radiculalgie se présentait comme une sciatique dans 246 cas $(93,5 \%)$ et une cruralgie dans 17 cas $(6,5 \%)$, soit respectivement de topographie L5 dans 210 cas $(79,8 \%)$, S1 dans 36 cas $(13,7 \%)$, L4 dans 14 cas $(5,3 \%)$ et L3 dans 3 cas $(1,1 \%)$. L'intensité moyenne de la douleur à l'admission était de 8/10 selon l'échelle visuelle analogique (EVA), avec des extrêmes variant de 7 à 10/10. Il existait une claudication neurologique chez 235 patients $(63,3 \%)$ avec un périmètre de marche inférieur à 60 mètres chez 62 patients $(26,4 \%)$, entre 60 et 100 mètres chez 83 patients $(35,3 \%)$ et supérieur à 100 mètres chez 90 patients $(38,3 \%)$. A L'examen 
physique, 320 patients $(86,2 \%)$ présentaient un syndrome rachidien lombaire (Tableau II).

Tableau II : Tableau récapitulatif des patients ayant un syndrome rachidien lombaire

\begin{tabular}{lcc}
\hline & Effectifs & Fréquences (\%) \\
\hline Syndrome rachidien lombaire & $\mathbf{3 2 0}$ & $\mathbf{8 6 , 2}$ \\
Scarifications & 133 & 41,5 \\
Hyperlordose & 43 & 31,0 \\
Scoliose & 95 & 69,0 \\
Douleur à la palpation des épineuses & 273 & 85,0 \\
Déformation en marche d'escalier & 23 & 7,1 \\
Contracture musculaire paravertébrale & 37 & 11,5 \\
Inclinaison latérale droite et/ou gauche & 128 & 47,2 \\
Rotation droite et/ou gauche & 30 & 11,0 \\
Flexion/extension & 260 & 96,0 \\
Indice de Schöber* & & \\
$0-5 \mathrm{~cm}$ & 0 & 0 \\
$5-10 \mathrm{~cm}$ & 4 & 1,5 \\
$10-15 \mathrm{~cm}$ & 198 & 76,2 \\
\hline $15 \mathrm{~cm}$ & 58 & 22,3 \\
\hline Distance doigts-sol & & \\
\hline 0 & 258 & 99,2 \\
\hline 0 & 2 & 0,8 \\
\hline
\end{tabular}

*La moyenne de l'indice de Schöber était de $12 \pm 4,3 \mathrm{~cm}$.

Un syndrome radiculaire était objectivé chez 235 patients $(63,3 \%)$, de topographie sciatique chez 220 patients $(93,6 \%)$ et de topographie crurale chez 15 patients $(6,3 \%)$. L'amplitude moyenne du signe de Lasègue était de 50,4 \pm 20,2 degrés (Tableau III).

Tableau III : Tableau récapitulatif des patients présentant un syndrome radiculaire

\begin{tabular}{|lcc|}
\hline Syndrome radiculaire & Effectifs & Fréquences (\%) \\
\hline Unilatéral & 235 & 63,3 \\
Bilatéral & 123 & 52,4 \\
Sciatalgie & 112 & 47,6 \\
\hline L5 & & \\
S1 & 205 & 93,2 \\
\hline Cruralgie & 15 & 6,8 \\
\hline L3 & & \\
L4 & 5 & 33,3 \\
Signe de la sonnette & 10 & 66,7 \\
Signe de Lasègue & 160 & 68,0 \\
\hline 30 & 220 & 93,6 \\
\hline $30-45$ & 12 & 5,5 \\
$45-60$ & 38 & 17,2 \\
\hline 60 & 94 & 42,8 \\
Signe de Léri & 76 & 34,5 \\
\hline
\end{tabular}


Soixante-treize patients $(19,7 \%)$ présentaient un déficit neurologique. Parmi eux, 67 des troubles sensitifs $(91,8 \%) 13$ des troubles moteurs $(17,8 \%)$ et 2 avaient des troubles sphinctériens (2,7\%), (Tableau IV).

Tableau IV : Répartition des patients en fonction du déficit neurologique

\begin{tabular}{lcc}
\hline Déficit neurologique & Effectifs & Fréquences (\%) \\
\hline Troubles sphinctériens & 2 & 2,7 \\
\hline Troubles sensitifs & 67 & 91,8 \\
Hypoesthésie & 50 & 74,6 \\
Paresthésie & 17 & 25,4 \\
Anesthésie en selle & 0 & 0 \\
Troubles moteurs & 13 & 17,8 \\
FM > 3 & 12 & 92,3 \\
FM <3 & 1 & 7,7 \\
Atteinte distale & 13 & 100 \\
Atteinte globale & 0 & 0 \\
\hline
\end{tabular}

La localisation L4-L5 était la plus fréquente objectivée dans 270 cas $(72,8 \%)$ suivi de localisation $\mathrm{L} 5-\mathrm{S} 1$ et L3-L4 avec respectivement 59 cas $(15,9 \%)$ et 42 cas $(11,3 \%)$. Aucun patient ne présentait un SPL localisé aux étages L1-L2 et/ou L2-L3. Il s'agissait d'un antélisthésis dans 99\% des cas. Ceux qui avaient un rétrolisthésis ou un latérolisthésis représentaient chacun $0,5 \%$ des cas. Le SPL était, selon la classification de Meyerding, de grade I dans 265 cas $(71,4 \%)$ et de grade II dans 106 cas $(28,6 \%)$. Aucun SPL de grade III, IV ou V n'a été retrouvé. Le principal type de SPL, selon la classification de Wiltse, était le type III ou dégénératif (SD) retrouvé chez 340 patients $(91,6 \%)$. Chez 31 patients $(8,4 \%)$, il s'agissait d'un type II ou isthmique (SLI). Aucun cas de SPL dysplasique, traumatique ou pathologique n'a été objectivé.

\section{Discusion}

La place du spondylolisthésis dans la pathologie rachidienne mécanique en Afrique Sub-saharienne est mal définie. Il n'existe aucune étude en population sur la prévalence ou l'incidence du SPL à notre connaissance. Les données concernent surtout les populations blanche, asiatique et les esquimaux avec respectivement une prévalence de 5 à $6 \%, 9,6 \%$ et 15 à $54 \%$ (Abdoun A et al, 2001). Le spondylolisthésis apparait pourtant comme une affection fréquente en Afrique, au regard des données des séries hospitalières. Amadou et al au Togo (Amadou A et al, 2017), rapportait une fréquence de 9,93\% sur une population d'étude de 1943 cas, Ntsiba et al une fréquence de $15 \%$ sur 200 cas de lombalgie commune (Ntsiba H et al, 2009), enfin dans la présente série, elle était de 13,2\%. Le SPL intéresse autant l'homme que la femme avec une prédominance qui varie selon les séries (Amadou A et al, 
2017; Iba Ba J et al, 2006; Fojo T.B et al, 2018). Dans cette étude, la prédominance féminine traduit essentiellement la prépondérance féminine de la pathologie rachidienne lombaire mécanique en milieu rhumatologique au Congo, constat fait par Bileckot et al (Bileckot R et al, 1992) et Ntsiba et al (Ntsiba $\mathrm{H}$ et al, 2009). L'âge moyen de survenue du SPL, dans la présente étude, de 61,2 \pm 9,8 ans, parait tardif en comparaison aux séries de Amadou et al au Togo (Amadou A et al, 2017), de Iba Ba et al au Gabon (Iba Ba J et al, 2006), de Fojo et al au Cameroun (Fojo T.B et al, 2018) et Varlet et al en Côte d'Ivoire (Varlet $\mathrm{G}$ et al, 1997) avec respectivement 43 ans, 51,8 \pm 9 ans, $57,1 \pm 12,6$ ans et 52 ans de moyenne d'âge. Ces résultats suggèrent que le SPL est l'apanage de l'adulte d'âge mûr, voire de la personne âgée en Afrique Tropicale. Chez la personne âgée la prévalence du SPL augmente avec l'âge. Elle est estimée à $30 \%$ entre 65 et 69 ans et dépasse $39 \%$ au-delà de 80 ans (Denard P J et al, 2010). Aucun cas de SPL n'a été rapporté chez l'enfant, dans ces différentes séries, alors qu'il apparait fréquent chez l'enfant dans les séries européennes, de l'ordre de 4,4\% à 6 ans, de 5,2\% à 12 ans et de 5,6\% à 14 ans (Mac-Thiong J-M et al, 2011). Chez l'enfant, le SPL par lyse isthmique prédomine. Il résulte de facteurs mécaniques notamment la pratique sportive et de facteurs génétiques non encore élucidés (Dupont $\mathrm{P}$ et al, 2008). Il peut cependant rester asymptomatique très longtemps et ne se révéler qu'à l'âge adulte (Boisaubert B et al, 2001; Abdoun A et al, 2001). Ce mode de révélation tardif du SPL peut expliquer l'absence de cas colligés et donner une fausse impression d'absence de cas chez l'enfant, dans les séries africaines (Varlet G et al, 1997; Amadou A et al, 2017; Iba BA J et al, 2006; Fojo T.B et al, 2018). Cependant, il existe un biais de sélection dans les séries d'Afrique subsaharienne, lié au recrutement en milieu hospitalier adulte, sans tenir compte du milieu hospitalier pédiatrique. Ce qui rend toute conclusion difficile quant à la place du SPL chez l'enfant en Afrique.

En Afrique Sub-saharienne, la pratique d'une activité sportive est peu courante, comme en témoigne le chiffre peu élevé de sportif dans notre série (Boisaubert B et al, 2001). Les facteurs professionnels semblent être plus prépondérants dans la genèse du SPL. Dans notre série les femmes au foyer sont les plus touchées. Les activités de vie courante sont le plus souvent réalisées à la force des bras, entrainant une forte sollicitation du rachis lombaire. Dans la série de Amadou et al, ce sont les conducteurs, les cultivateurs et les menuisiers qui constituent les professions à risque, professions toutes à forte sollicitation mécanique pour le rachis (Amadou A et al, 2017). Chen et al ont montré, dans leur série sur les taximen, que le type d'activité professionnelle était corrélé au risque de développer un SPL (Chen J C et al, 2004).

Dans les séries africaines, la lombalgie et la lomboradiculalgie sont les modes de révélation les plus fréquents du SPL. La radiculalgie est plus souvent 
unilatérale, de topographie sciatique notamment L5 (Amadou A et al, 2017; Iba BA J et al, 2006; Fojo T.B et al, 2018), posant ainsi le problème de diagnostic différentiel avec une discopathie (Abdoun A et al, 2001). La claudication neurologique intermittente est également fréquente, retrouvée dans $80 \%$ des cas dans la série de Varlet et al (Varlet G et al, 1997) et dans $63,3 \%$ des cas dans notre série. Cette claudication se manifeste par une limitation du périmètre de marche, soit douloureuse par le déclenchement de la radiculalgie, soit par fatigabilité des membres inférieurs (Abdoun A et al, 2001). L'examen physique retrouve de façon systématique un syndrome rachidien lombaire, volontiers facettaire postérieur. Le syndrome radiculaire parait moins fréquent et est souvent confondu avec le syndrome de rétraction douloureuse des ischio-jambiers, particulièrement fréquent au cours du SPL, responsable de la raideur constatée lors de la mesure de la distance doigt-sol et surtout d'un pseudo Lasègue (Vialle R et al, 2007). Ce pseudo- Lasègue peut expliquer l'amplitude moyenne ample du signe de Lasègue (50,4 20,2 degrés) retrouvée dans notre série. Le syndrome neurologique déficitaire est peu fréquent au cours du SPL (19,7\% dans notre série), passant parfois inaperçu. Il prédomine en distalité, le plus souvent de type parésiant. Les troubles sphinctériens sont rares et difficiles à mettre en évidence, exceptés dans le SPL de haut grade avec compression de la queue de cheval (Iba BA J et al, 2006). Tous ces signes cliniques sont malheureusement non spécifiques et obligent la réalisation d'une imagerie pour le diagnostic de certitude du SPL. Le meilleur examen d'imagerie reste la radiographie standard du rachis lombaire en incidence de face, de profil et de trois quart (Vialle R et al, 2007; Haun D w et al, 2005). Elle est suffisante pour objectiver le déplacement antérieur vertébral, en préciser le type et apprécier le grade selon la classification de Meyerding. Elle ne permet pas cependant l'analyse des articulations inter apophysaires postérieures, ni le retentissement sur les structures nerveuses intra canalaires, d'où l'intérêt de l'imagerie en coupe (TDM et IRM) (Vialle R et al, 2007; Haun D W et al, 2005). L'imagerie en coupe reste peu prescrite dans les séries africaines $(21,0 \%$ dans la présente série), la TDM étant plus souvent indiquée que l'IRM. En Afrique Subsaharienne, le coup élevé et l'insuffisance du plateau technique limitent fortement leur prescription. Que ce soit dans les séries occidentales ou africaines, le SPL siège principalement aux étages L4-L5 et L5-S1. Les sièges hauts (L1-L2 ; L2-L3) sont rarement décrits et uniquement rapportés dans les séries occidentales (Gille O et al, 2014). En Afrique sub-saharienne, le type dégénératif est prédominant. Les facteurs génétiques et environnementaux (stress physique et pratique sportive) joueraient un rôle prépondérant dans la pathogénie du SPL et expliqueraient ses variations selon la race (Haun D W et al, 2005). Les grades I et II s'avèrent être les plus décrits dans la littérature, ils prédominent chez l'adulte comme chez la personne âgée. Les SPL de haut 
grade sont exceptionnels, rapportés dans les SPL par lyse isthmique chez le jeune sportif de haut niveau (Bollini $\mathrm{G}$ et al, 2010).

\section{Conclusion}

Au Congo, Le SPL apparait comme une affection fréquente en milieu hospitalier. Le SPL prédomine chez l'adulte d'âge mûr, surtout de sexe féminin et s'exprime par une lombalgie et/ou une lomboradiculalgie mécanique, parfois parésiante. La radiographie standard est suffisante pour poser le diagnostic. Le SPL siège exclusivement aux étages L4-L5 et L5-S1 du rachis lombaire. L'étiologie dégénérative en est la principale cause. L'imagerie en coupe n'est pas systématique, indiquée en cas d'échec du traitement médical ou de déficit neurologique patent.

Conflits d'intérêt : les auteurs ne déclarent aucun conflit d'intérêt.

\section{References:}

1. Herbiniaux G. (1782).Traité sur divers accouchements laborieux et sur des polypes de la matrice. Bruxelles.

2. Vital J M, Pedram M. (2005). Spondylolisthésis par lyse isthmique. EMC- Rhumatologie Orthopédie; 2: 125-50.

3. Meyerding H W. (1932). Spondylolisthesis. Surgery, Gynecology and Obstetrics; 54: 371-7.

4. Wiltse L L, Newman PH, Macnab I. (1976). Classification of Spondylolysis and Spondylolisthesis. Clinical Orthopaedics and Related Research; 117: 23-9.

5. Boisaubert B, Descamps H, Beaupere G D, Hecquet J, Marty C, Montigny J P et Zujovic J. (2001). Spondylolisthésis: Morphotype sacré et lyse isthmique. Rachis; 13(2): 123-34.

6. Dufor X, Barette G, Ghossoud P et Loriferne A. (2013). Spondylolisthésis et rééducation fonctionnelle. Kinestither Review; 13(139): 13-9.

7. Varlet G, Boukassa L, Ba Zézé V. (1997). Le spondylolisthésis au CHU d'Abidjan (Côte d'Ivoire). Revue des cas et perspectives. Annales de Médecine; 26: 339-49.

8. Amadou A, Sonhaye L, Kombaté D, Tchaou M, WataraG, N'timon B, Adje $\neg$ nou KE, N'dakena K. (2017). Profil radiologique des spondylolisthésis lombaires de l'adulte à LOME. Revue Africaine et Malgache de Recherche Scientifique/Sciences de la Santé; 5 (1): 1004.

9. Iba BA J, Mwanyombet L, Nkoghe D, Assengone Zeh Y, Chouteau PY. Loembe P-M. (2006). Traitement chirurgical du Spondylolisthésis au Gabon. Neurochirurgie; 52 (4): 339-46. 
10. Ntsiba H, Makosso E. (2009). La lombalgie commune à propos de 200 cas observés dans le service de Rhumatologie du CHU de Brazzaville. Médecine d'Afrique Noire; 56 (4): 226-30.

11. Abdoun A, Leonard J C, Plais P Y. (2001). Lyse isthmique spondylolisthéisis clinique, diagnostic, prise en charge. Rachis; 13(2) :149-56.

12. Fojo T B, Doualla M S, Ongolo Z P, Ngoufack C, Kemta L F, Ngandeu-Singwe M. (2018). Spondylolisthésis lombaire : étude monocentrique à l'Hôpital Régional de Bertoua. Journal Africain d'Imagerie Médicale; 10 (1): 19-20.

13. Bileckot R, Ntsiba H, Mbongo J A, Masson Ch, Bregeon Ch. (1992). Les affections rhumatismales observées en milieu hospitalier au Congo. La Semaine des Hôpitaux de Paris; 68: 282-5.

14. Denard P J, Holton K F, Miller J, Fink H A, Kado D M., Yoo J U and Marshall L M. (2010). Lumbar spondylolisthesis among elderly men: prevalence, correlates and progression. Spine (Phila Pa 1976); 35(10): 1072-8.

15. Mac-Thiong J-M et Labelle M. (2011). Classification et prise en charge du spondylolisthésis lombosacré chez les jeunes athlètes. Journal de Traumatologie du sport; 28 (2) :90-8.

16. Dupont P. (2008). spondylolisthésis et sport. Lettre de Médecine Physique et Réadaptative; 24: 152-6.

17. De Souto Barreto P. (2013). Why are we failing to promote physical activity globally? Bulletin of the World Health Organization; 91: 390390A

18. Chen J C, Chan W P, Katz J N, Chang W P, Christiani D C. (2004). Occupational and personal factors associated with acquired lumbar spondylolisthesis of urban taxi drivers. Occupational and Environmental Medicine; 61 (12): 992-8.

19. Vialle R, Benoist M. (2007). High-grade lumbosacral spondylolosthesis in children and adolescents: Patogenesis, morphological analysis and thérapeutic strategy. Joint Bone Spine; 74(5): 414-7.

20. Haun D w, kettner N. (2005). Spondylolysis and spondylolisthesis : a narrative review of etiologie, diagnosis and conservative management. Journal of Chiropratic Medecine; 4: 206-1

21. Gille O, Challier V, Parent H, Cavagna R, Poignard A, Faline A, Fuentes S, Ricart O, Ferrero E, Ould Slimane M. (2014). Le spondylolisthésis lombaire dégénératif. Cohorte de 670 patients et proposition d'une nouvelle classification. Revue de Chirurgie Orthopédique et Traumatologie; 100 (6): 311-5. 
22. Bollini G, Blondel B, Launay F, Viehweger E, Jacopin S, Jouve J-L. (2010). Spondylolisthesis de haut grade : biomécanique et proposition thérapeutique. E-mémoires de l'Académie Nationale de Chirurgie; 10 (3): 22-6. 